\title{
Biliary tract abnormalities associated with duodenal atresia
}

\author{
IAN S. REID \\ From the Children's Medical Research Foundation, Royal Alexandra Hospital for Children, Camperdown, N.S.W.; and \\ the Department of Surgery, University of Sydney, Australia
}

Reid, I. S. (1973). Archives of Disease in Childhood, 48, 952. Biliary tract abnormalities associated with duodenal atresia. The embryology of the duodenum and biliary tracts makes understandable those developmental abnormalities of the biliary tract that occur in association with duodenal atresia. In a series of 167 patients with intrinsic duodenal obstruction, a number also showed anomalies of the distal common bile duct, and these form the subject of this communication.

The clinical importance of stenosis of the distal common bile duct seen in duodenal atresia and stenosis is stressed; operative intervention in the region may result in complete biliary obstruction. 3 patients in the series had agenesis of the gallbladder in association with duodenal atresia, an association not previously recognized.

A developmental study of the duodenum and the pancreatic and biliary ducts is necessary to understand the abnormalities commonly seen in the region. Gourevitch (1971) and others have shown duplication and stenosis of the distal common bile duct in association with duodenal atresia. Included in this paper are three examples of aplasia of the gallbladder in patients with duodenal atresia. All these associations with duodenal atresia are predictable from the known embryology. Though no cases of choledochal cyst were found in the present series of duodenal atresia, with a knowledge of embryology this association could be anticipated.

\section{Embryology}

The Viennese anatomist Tandler, in 1900 (quoted by Boyden, Cope, and Bill, 1967), postulated the theury of epithelial hyperplasia and recanalization by vacuolation as the basis of development of the duodenal lumen. Debate on this development over the next 60 years came from observers such as Forssner (1907, quoted by Boyden et al., 1967), Johnson (1910), and Lynn and Espinas (1959). By 1960 it was generally accepted that the theory explained development of the duodenum especially in the lumen of the second part. The usual types of duodenal atresia as seen at the second part could be adequately explained as aberrations during the

Received 11 June 1973. development as described by Tandler. It was stressed that a different process occurred in the jejunum and ileum, and Barnard and Louw (1956), in showing the vascular aetiology of jejunal and ileal atresias, rightly excluded the duodenum. However, any theory must explain not only the event of duodenal atresia, but also the other complex abnormalities that occur in this region. Sutton (1889) established the principle that 'congential obstruction and narrowing of the alimentary canal are always found in the situation of embryological events'. Few parts of the alimentary tract would have greater claim to classification as such a 'situation' than the second part of the duodenum. Boyden et al. (1967), in discussing the abnormalities which may occur at the entrance of the common bile duct and pancreatic ducts, stated that they were the result of an embryological 'traffic jam'. They showed that during development two blindly ending channels appear in the duodenum at the stage of vacuolization and at the same time two channels also appear in the developing biliary system. Each of the latter join up separately with the developing duodenal channels. If some accident affected the 'embryological events' at this stage of development, aberrations of the lower biliary tract could be expected in association with duodenal atresia.

The sequence of embryological events in the development of the area is shown in the Table. The foregut appears very early and rapid development occurs at its junction with the midgut. The 


\section{Biliary tract abnormalities associated with duodenal atresia}

TABLE

Sequence of events in development of duodenum, pancreas, and biliary tract

\begin{tabular}{|c|c|c|}
\hline $\begin{array}{l}\text { Fetal age } \\
\quad(\mathrm{dy})\end{array}$ & $\begin{array}{l}\text { Fetal length } \\
\text { (crown rump) } \\
\quad(\mathrm{mm})\end{array}$ & Alimentary tract development \\
\hline $\begin{array}{l}22 \\
26\end{array}$ & $\begin{array}{l}2 \\
4\end{array}$ & $\begin{array}{l}\text { Foregut can be distinguished } \\
\text { Hepaticobiliary and dorsal pancreas } \\
\text { buds appear }\end{array}$ \\
\hline 38 & 11 & $\begin{array}{l}\text { Gallbladder, bile ducts, and hepatic } \\
\text { ducts are distinguishable }\end{array}$ \\
\hline $\begin{array}{l}43 \\
49\end{array}$ & $\begin{array}{l}17 \\
28\end{array}$ & $\begin{array}{l}\text { Duodenal lumen obliterated } \\
\text { Fusion of dorsal and ventral } \\
\text { pancreas; bile ducts and } \\
\text { duodenum recanalized }\end{array}$ \\
\hline $\begin{array}{r}84 \\
112\end{array}$ & $\begin{array}{r}88 \\
140\end{array}$ & $\begin{array}{l}\text { Bile present } \\
\text { Meconium present in gut }\end{array}$ \\
\hline
\end{tabular}

terminal portion of the foregut and the most cephalic portion of the midgut grow rapidly to form a loop which is the duodenum on its dorsal mesentery (mesoduodenum). The apex of this loop represents the junction between the embryonic foregut and midgut, and from its endodermal lining grows out the hepaticobiliary and pancreatic buds. These appear as early as the 26th day or $4 \mathrm{~mm}$ stage, and within the next 12 days the biliary system including the gallbladder can be distinguished. The terminal bile ducts grow out into the mesenchymal tissue of the septum transversum which will produce the fibrous architecture of the liver. The endodermal cells will give rise to the hepatic cells, but these do not appear to produce bile until much later (84 days), by which time two significant processes have been completed: (1) the epithelial hyperplasia and recanalization by vacuolation of the duodenum and common bile duct, and (2) the rotation and fusion of the ventral and dorsal pancreases.

Knowledge of this embryological sequence therefore indicates that if atresia of the bile ducts were to occur as a developmental abnormality, this would have occurred before the production of bile and meconium. The patients seen with biliary atresia in clinical practice have bile in the meconium and it is unlikely the condition is due to failure to recanalize after epithelial hyperplasia. Such a bile duct atresia would probably be fatal to the fetus. However, bile duct stenosis would be possible and in fact likely if atresia or stenosis of the duodenum occurs due to some aberration of the formation of its lumen by epithelial hyperplasia and recanalization.

\section{Material and methods}

The records of patients with intrinsic duodenal obstructions at the Royal Children's Hospital, Melbourne (88 cases), and the Royal Alexandra Hospital for
Children, Sydney (79 cases), were examined. There were thus 167 patients, and necropsy reports were available for 81 of the 95 children who died. The series included 10 patients whose necropsy specimens were dissected (H. R. Noblett and C. Paton, personal communication, 1972). In addition to these 10 patients, 5 others were regarded as having obstruction to the common bile duct at postmortem examination. The case histories of these 5 patients, together with 3 others who had aplasia of the gallbladder and one from the Royal Alexandra Hospital who had bifurcation of the common bile duct, are presented below. The necropsy specimen from Case 8 was examined in detail by the author.

Case 1. This infant was admitted at the age of 8 hours with multiple congenital deformities including tracheooesophageal fistula, duodenal atresia, annular pancreas, incomplete rotation of gut, partial situs inversus, imperforate anus, cardiovascular anomalies, gross urogenital abnormalities, and arthrogryposis. No specific treatment was given and the child died $1 \frac{1}{2}$ hours after admission. At necropsy the relevant findings were: 'gallbladder was small and contained green bile. However, it was impossible to express this bile through the cystic duct nor was it possible, on opening the gallbladder, to pass a fine probe more than $5 \mathrm{~mm}$ down the cystic duct. The common bile duct was seen to enter the region of the atretic duodenum surrounded by pancreas. It was impossible to pass a probe through; however, the large bowel contained meconium.'

Histology of the liver showed 'widespread vacuolation of the liver parenchyma presumably due to fatty change. Lobules are formed but both these and the portal tracts are more difficult to identify than usual'.

Case 2. This infant had a duodeno-duodenostomy performed on the 3 rd day of life and died on the 11 th day. The relevant section of the necropsy report was 'the gallbladder was distended with bile. The cystic, hepatic, and common bile ducts were markedly dilated with bile. There was complete blockage of the common bile duct distally'. The duodeno-duodenostomy anastomosis was patent. Histology report was as follows, 'liver: early stage of obstructive biliary cirrhosis with retained bile in ducts and ductules and slight thickening and intralobular extension of the portal tracts. Pancreas: evidence of ductal obstruction'.

Case 3. A duodenojejunostomy was performed on this child on the 4th day of life and he died on the 23rd day. After operation the child passed brown motions initially, but these later were described as yellow curds. At necropsy the gallbladder was 'small, empty, and not bile stained'. No comment was made on the bile ducts. However, the serum bilirubin rose after operation to a total of $7.4 \mathrm{mg} / 100 \mathrm{ml}$, with direct $5.9 \mathrm{mg} / 100 \mathrm{ml}$ and indirect $1.5 \mathrm{mg} / 100 \mathrm{ml}$. Histology of the liver at necropsy showed 'fibrosis of portal tracts with slight dilatation of the larger bile ducts and an early increase in ductules at the periphery of the lobules. There is 
marked bile stasis principally towards the centres of the lobules. Pancreas: ascending infection of the pancreatic ducts with patchy acute interstitial inflammation'.

Case 4. This child with Down's syndrome became jaundiced and developed signs of duodenal obstruction on the second day of life. She developed convulsions and died shortly after admission.

At necropsy examination there was an area of duodenal atresia in the second part at the entrance of the pancreatic and common bile ducts. The gallbladder was normal but a probe could not be passed along the common bile duct and there was no obvious ampulla of Vater. Bile in the gallbladder could not be expelled along the common bile duct. There was no mention of whether or not meconium was present in the gut. Histology of the liver revealed normal architecture with congested vessels. The bile ducts were dilated and filled with granular bile pigment. The pancreas appeared normal histologically.

Case 5. This infant was admitted on the 3rd day of life. She was 'slightly jaundiced' on admission. The next day laparotomy was performed and a complete septum in the second part of the duodenum was excised and a transanastomatic tube inserted. A few days after operation the jaundice 'increased considerably' and the serum bilirubin rose to a total level of $27.2 \mathrm{mg} / 100 \mathrm{ml}$, with direct $10.5 \mathrm{mg} / 100 \mathrm{ml}$ and indirect of $16.7 \mathrm{mg} / 100$ $\mathrm{ml}$. The child died on the 5 th day after operation.

At necropsy examination the duodenal suture line had broken down with resulting intraperitoneal abscess formation. No ampulla of Vater could be found. The common bile duct, cystic duct, and gallbladder were dilated and contained bile. The common bile duct 'passed down behind the distal duodenal stump, but it was found to end blindly close to or actually within the wall of the duodenum.' Histology of the liver was reported as showing 'extreme congestion, cells vacuolated, a few are degenerated. Extensive fatty change in most of the liver cells'. The pancreas showed 'some acini are dilated. Lobules separated by wide swathes of oedematous connective tissue. No appreciable intralobular connective tissue present'.

Case 6. The records noted 'prenatal influences normal'. This child was found to have oesophageal atresia with tracheo-oesophageal fistula and duodenal obstruction at $x$-ray examination on the second day of life; and on the same day the fistula was divided and gastrostomy and duodenojejunostomy were performed. The baby died on the 3rd day after operation. At necropsy examination the distal stump of the tracheooesophageal fistula had broken down. The duodenojejunostomy was intact with an adequate lumen. 'Marked stenosis of a short segment of the second part of the duodenum was present, the gut lumen in the stenosed portion being pin-hole size, but allowing fluid to be squeezed through. The distal duodenum and first part of the jejunum were collapsed, and their walls deeply bile stained'. The extrahepatic tree was described as follows, 'the gallbladder was completely absent, but the common bile duct was patent. It was difficult to determine exactly where this common duct entered the duodenum, but it appeared to do so just distal to the stenosed part of the duodenum'. The only other congenital abnormality noted was an $8 \mathrm{~mm}$ atrial septal defect.

Case 7. This child was born of a 22-year-old primipara. No details of the health or antenatal management of the mother were available. The child had severe multiple abnormalities and was given only supportive care and died at the age of 10 hours. In addition to the duodenal and bile tract abnormalities the child had oesophageal atresia with tracheo-oesophageal fistula, anorectal atresia, bilateral talipes, hypoplastic lungs, absent right ureter and kidney, multicystic left kidney, bifid scrotum, and congenital dislocation of the hips.

There were two umbilical arteries. The stomach was collapsed and there was a nodule of ectopic pancreatic tissue on the wall of the pylorus. The proximal duodenum was not dilated and ended blindly in the second part of the level of the head of the pancreas. The gap between proximal and distal parts of the duodenum was $6 \mathrm{~mm}$. The distal duodenum, jejunum, and ileum were collapsed.

The liver was of the normal shape and texture, weighing $63 \mathrm{~g}$. Histologically there were scattered foci of haemopoiesis, but otherwise it was normal. The hepatic and portal veins were unremarkable. The gallbladder and cystic duct were absent, but the bile ducts were present and patent.

Case 8. This infant was $1 \cdot 75 \mathrm{~kg}$ at birth and was regarded as 34 weeks' gestation. His mother was unmarried and was given sulphadimidine $0.5 \mathrm{~g}$ twice daily throughout the pregnancy prophylactically for rheumatic fever as she was allergic to penicillin. In addition, she was given Debendox tablets and told to take two tablets as required during the early weeks of pregnancy when vomiting was a problem. She had 'influenza' during the 2nd month of pregnancy and otitis media in the 5 th month when she was treated with erythromycin.

At birth it was noted that the baby had only one umbilical artery. The child vomited bile-stained fluid on the third day of life and was admitted to hospital where abdominal $x$-rays showed the typical 'double bubble' of duodenal atresia. Operation was deferred until the 5th day of life because of the child's poor condition on admission. At operation atresia of the second part of the duodenum was found and duodenoduodenostomy and gastrostomy were performed. The child became cyanosed and collapsed on the 3rd postoperative day and failed to respond to resuscitation.

At necropsy examination there was bilateral pulmonary oedema with patchy consolidation of the lungs.

The duodeno-duodenostomy was intact and the stoma adequate. The atresia began $2.5 \mathrm{~cm}$ from the pylorus and there was a complete gap of $3 \mathrm{~mm}$. Pancreas was present in the base of the gap but there was no 'annular 
pancreas'. The distal duodenum was bile stained and began as a dome-shaped structure with an external diameter of $5 \mathrm{~mm}$. As seen in Fig. 1 a large funnel-
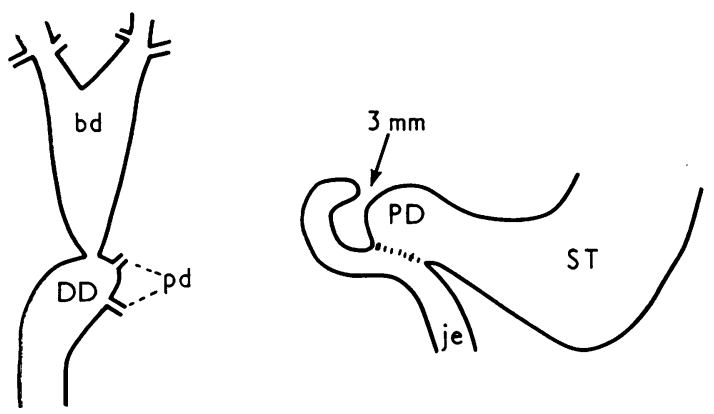

FIG. 1.-Case 8. Necropsy findings of the biliary tract and duodenal region. $D D$, distal duodenum; $P D$, proximal duodenum; ST, stomach; bd, bile duct; pd, pancreatic ducts; je, jejunum.

shaped bile duct passed from the most proximal part of the distal duodenum up in the lesser omentum to the porta hepatis where it divided into two hepatic ducts which passed into the right and left lobes of the liver. There was no gallbladder or cystic duct. No duct was found between the biliary tracts and the proximal duodenum. The large bile duct was $2 \mathrm{~mm}$ in external diameter at its junction with the duodenum; it then enlarged to a maximum diameter of $6 \mathrm{~mm}$ just before it divided into right and left hepatic ducts. Two pancreatic ducts were seen passing to the duodenum; one in close association with the entrance of the large bile duct and another $2 \mathrm{~mm}$ distal to the entrance.

Fig. 2 is an $x$-ray of the biliary tracts after dye was injected into the large bile duct. Considerable pressure was required to force dye into the duodenum because of narrowing of the duct at its entrance to the distal duodenum. A normal intrahepatic biliary tree was shown but no intrahepatic gallbladder. No dye passed into the proximal duodenal segment.

There was a Meckel's diverticulum and a small diverticulum on the antimesenteric border of the jejunum. There were no other abnormalities found at necropsy examination.

The liver histologically showed cholestasis, principally centrolobular in distribution. The bile duct system was normal. The pancreas was histologically normal.

Case 9. This 2-day-old child was admitted to hospital in 1936, with vomiting, gastric dilatation, and visible peristalsis. An enema was given but no meconium obtained. He vomited 'two large quantities of blood stained fluid after admission'. The child died 2 days after admission. No other clinical information is available.

A necropsy report states the child was jaundiced. The stomach was greatly dilated and occupied 'the whole of the epigastric, umbilical, and left hypochondrial compartments'. The dilatation extended into the proximal duodenum just beyond the entrance of the common bile duct. Fig. 3 is a photograph of the organs which are preserved in the museum of the hospital's Institute of Pathology. As can be seen from the specimen, the common bile duct gives off a small duct going to the distal duodenum while the main duct passes

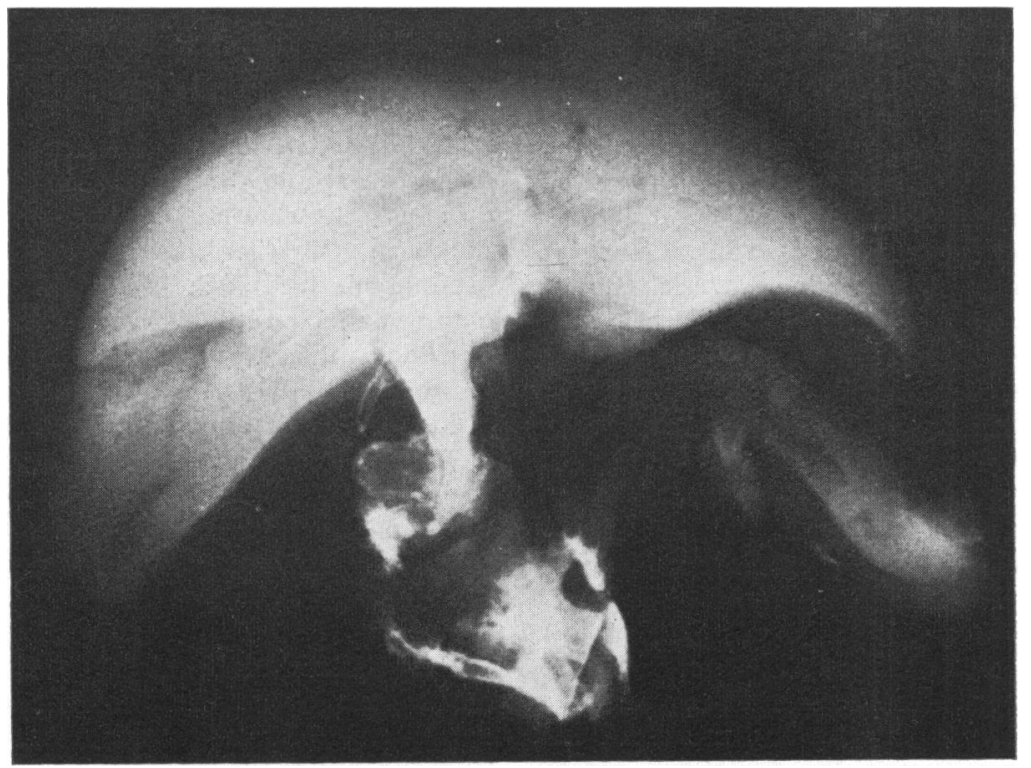

FIG. 2.-Case 8. Postmortem x-ray of biliary tracts after injection of radio-opaque dye (see text). 


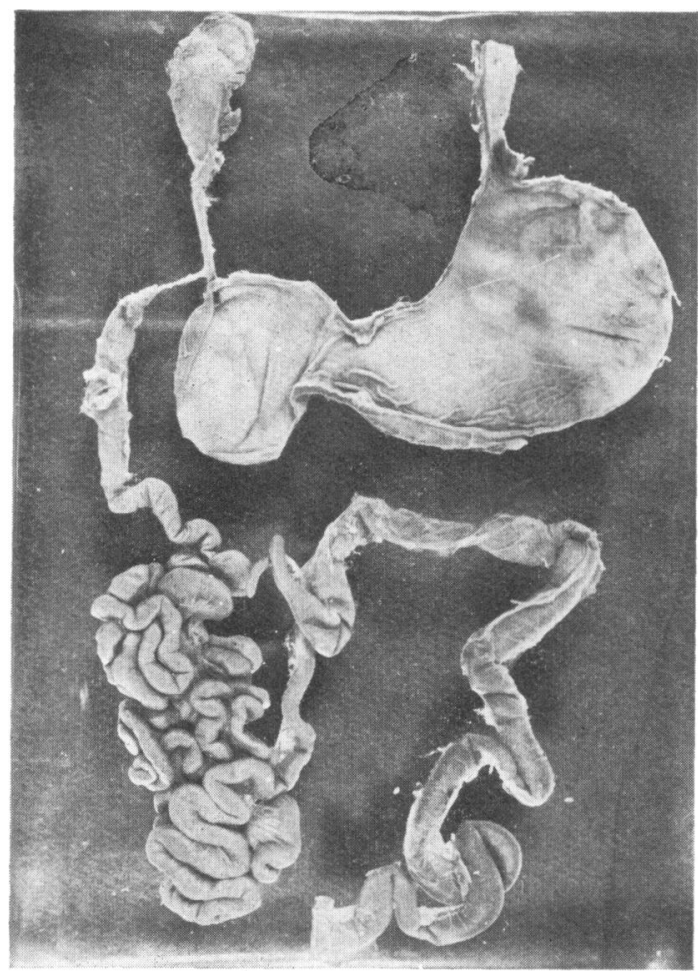

FIg. 3.-Case 9. Photograph of a specimen in the museum of the Institute of Pathology, Royal Alexandra Hospital for Children, Sydney, from a necropsy examination of a child who died from duodenal atresia in 1936 (see text).

to the duodenum proximal to the atretic duodenal segment.

\section{Discussion}

Case 9 is of historical interest and is yet another example of partial duplication of the distal common bile duct in association with duodenal atresia. Similar cases were cited by Boyden et al. (1967) as having been reported by Karpa (1906) and Katz (1930). Further examples of pancreatic and bile duct abnormalities in association with duodenal atresia have been shown by Gourevitch (1971), who concluded that the atretic segment of duodenum was an abnormally situated bile duct. Noblett and Paton dissected 10 necropsy specimens of duodenal atresia at the Royal Children's Hospital, Melbourne(H. R. Noblett and C. Paton, personal communication, 1972), and found that in 5 the common bile duct divided at a $\mathrm{T}$-junction, the proximal end being covered by a flap valve of duodenal mucosa and the distal end opening at an ampulla with the dorsal and ventral pancreatic duct passing into the body and head of the pancreas. In the other 5 cases variations of this pattern occurred.

Those observers also pointed out that in the 10 specimens the bile ducts outside the area of the atretic duodenum were normal. However, if such lower biliary tract abnormalities occur, aberrations of other parts of the developing biliary tract, such as the gallbladder, could be anticipated. Cases 6, 7, and 8 are examples of agenesis of the gallbladder in association with duodenal atresia. Congenital absence of the gallbladder is rare. Cases 7 and 8 occurred within the last 3 years. Necropsy records of the two hospitals over that same period revealed only one other example of absent gallbladder in a child who died at the age of 4 weeks after corrective surgery for total anomalous pulmonary venous drainage. Monroe (1959) circularized 799 pathologists and reported 117 cases of absent gallbladder from $1,352,000$ necropsies, giving an incidence of approximately 1 in 7500 . The presence of 3 cases in association with duodenal atresia in our series is therefore significant. Monroe found the following associated duodenal abnormalities: diverticulum of the duodenum 2, 'dystrophy' of the duodenum, and annular pancreas 2. Monroe was actually reporting absence of the gallbladder and not agenesis. Frey, Bizer, and Ernst (1967) reviewed the published reports in English, including Monroe's article, and used strict criteria to exclude unproven cases and absent gallbladder associated with biliary atresia or resulting from inflammatory necrosis such as may occur in biliary calculi. They concluded there were only 56 proven cases of gallbladder agenesis published in English up to 1967.

The gallbladder is distinguishable at 38 days of fetal life and the duodenal lumen is obliterated at 43 days. It would therefore be expected that an accident contributing to the dual anomalies of duodenal atresia and absence of gallbladder should occur about this time. Lenz (1963), in reporting on children affected by thalidomide in Europe, showed that the gallbladder or duodenum was involved in 8 of these children. Absence of the gallbladder occurred with a normal duodenum in 3 children. In 3 other children the duodenum was affected and the gallbladder spared. In 2 further children duodenal atresia and gallbladder aplasia occurred together. This group of 8 children included one set of twins; one twin had duodenal atresia associated with aplasia of the gallbladder, the other had aplasia of the gallbladder. Lenz (1963) also suggested that the lesions of duodenal atresia and gallbladder aplasia resulted from the administration of the drug from the 40th to the 45 th day after the last menstrual period. In our Case 8, two drugs were taken during 
this period. Debendox (dicyclomine $\mathrm{HCl}$, doxylamine succinate, pyridoxine) is commonly given for nausea and vomiting of pregnancy and is generally regarded as having no effect on the fetus. Smithells (1966) states that malformations have been produced in experimental animals by the administration of some of the sulphonamides, but that there is no evidence to suggest they are teratogenic in man. Probably more significant in Case 8 was the febrile illness described as 'influenza' during the second month of pregnancy.

In considering obstruction to the lower common bile duct as indicated in Cases 1 to 5, Gourevitch (1971) has already drawn attention to narrowing of the common bile duct in infants with duodenal atresia. Such narrowing is probably so common as to be usual if the atresia is in the region of the duct openings; however, in our 5 cases examined at necropsy, stenosis of the duct was so obvious as to be especially mentioned by the pathologists concerned.

In Cases 2, 3, and 5, operative intervention had obscured the original nature of the obstruction to the distal common bile duct, while Cases 1 and 4 did not have operation. The latter two were early in the series and no extensive dissection was carried out to ascertain clearly the pattern of the bile ducts as was done in later cases (H. R. Noblett and C. Paton, personal communication, 1972). In Case 1 the pathologist pointed out that normal meconium was present in the large bowel and concluded the obstruction to the distal common bile duct must have been stenosis rather than atresia. In Case 4 there was no mention in the clinical or postmortem notes whether normal meconium was passed or was present in the bowel at necropsy. The histology of the liver and pancreas did not suggest chronic obstructive jaundice.

In the postoperative cases it was even more difficult to state the nature of the biliary obstruction at birth. However, there is evidence that total biliary obstruction followed operation. Cases 2 and 3 passed normal meconium before operation and brown stools for 3 or 4 days after operation, after which the stools became pale. In Case 3 the direct serum bilirubin also suggested obstruction as it increased after operation. In Case 5, where more extensive surgery was performed at the site of the bile ducts, the evidence was even more convincing that operative intervention precipitated total biliary obstruction. Though slight jaundice was present on admission, the jaundice increased considerably and the direct serum bilirubin rose after operation.

These 3 operated cases suggest that operative oedema may precipitate complete obstruction in a stenotic distal common bile duct and should warn against unnecessary dissection at operation to define the nature of bile duct anomalies. Case 5 was complicated by perforation of the suture line, but should also serve as a warning against excision of a septum at the site of entrance of the bile duct.

I am grateful to Dr. R. D. K. Reye, Director of the Institute of Pathology, Royal Alexandra Hospital for Children, for permission to photograph the museum specimen in Fig. 3, and for permission to make a detailed postmorten examination of Case 8.

REFERENCES

Barnard, C. N., and Louw, J. H. (1956). The genesis of intestinal atresia. Minnesota Medicine, 39, 745.

Boyden, E. A., Cope, J. G., and Bill, A. H. (1967). Anatomy and embryology of congenital intrinsic obstruction of the duodenum. American Fournal of Surgery, 114, 190.

Frey, C., Bizer, L., and Ernst, C. (1967). Agenesis of the gallbladder. American fournal of Surgery, 114, 917.

Gourevitch, A. (1971). Duodenal atresia in the newborn. Annals of the Royal College of Surgeons of England, 48, 141.

Johnson, F. P. (1910). The development of the mucous membrane of the oesophagus, stomach and small intestine in the human embryo. American fournal of Anatomy, 10, 521 .

Lenz, W. (1963). Chemicals and malformations in man. Proceedings of the Second International Conference on Congenital Malformations, p. 263. International Medical Congress, New York.

Lynn, H. B., and Espinas, E. E. (1959). Intestinal atresia. Archives of Surgery, 79, 357.

Monroe, S. E. (1959). Congenital absence of the gallbladder. fournal of the International College of Surgeons, 32, 369.

Smithells, R. W. (1966). Drugs and human malformations; antibiotics. In Advances in Teratology, Vol. 1, p. 270. Ed. by D. H. M. Woollam. Logos Press, London.

Sutton, J. B. (1889). Imperforate ileum. American fournal of the Medical Sciences, 88, 457.

Correspondence to Mr. I. S. Reid, Children's Medical Research Foundation, P.O. Box 61, Camperdown N.S.W. 2050, Australia. 